\title{
A CAPACITIVE SENSOR FOR HUMAN-ROBOT INTERACTION
}

\author{
SVACO, M[arko]; BASIC, D[enis]; SEKORANJA, B[ojan] \& JERBIC, B[ojan]
}

\begin{abstract}
Human robot interaction is an issue mostly oriented toward service robotics but recently has a significant impact in the field of assembly applications. Assembly is a highly complex field where technical systems lack sophisticated human manipulation skills and possibilities. A simple capacitive sensor has been developed for aiding the human-robot interaction in industrial applications. The capacitive sensor consists of rubber foam with an embedded highly flexible metal mesh. The sensor is connected to an Arduino controller and mounted on the robot. When the human operator is in contact or in close vicinity of the robot the change of capacitance is detected. The Arduino controller sends messages through TCP/IP protocol to a FANUC LR 200iC 5L articulated robot arm controlling unit. The robot responds based on the input and is able to reduce speed or stop current movement and operation. A prototype sensor is described in detail and test applications are presented.
\end{abstract}

Keywords: Human-robot interaction, Capacitive sensor, Arduino microcontroller

\section{INTRODUCTION}

Today, flexible and autonomous assembly systems consist of various machines, robots and other equipment delegated within different architectures. Self organization [1] in evolvable assembly systems is a paradigm where agents organize themselves to provide functional behavior and effectively accomplish a certain assembly task. Self-awareness is another growing research direction where industrial systems should be quickly reconfigurable and utilize the plug-and-play approach, avoid time-consuming reprogramming and be more resistant to perturbation [2]. In [3] a probabilistic approach for robot group control is developed. This method of governing complex system behavior has grounds in descriptive logic and Bayesian Networks. A multiagent robotic assembly system aided by service oriented architecture is presented in [4]. Mentioned systems are capable of reconfiguring their current state and behavior based on different production scenarios, dynamic and unpredicted changes. A complex robotic assembly system is able to perceive the environment using vision systems, force-torque sensors and other sensory equipment.

In certain cases where complexity of information or application exceeds system ability delays or failures occur. Furthermore information acquired by sensors is sometimes inadequate for properly resolving an unpredicted situation. Vision systems are confined with technical restrictions such as frame rate, resolution, color depth and limited computational power. The perception capabilities of a technical assembly system are confined and therefore possess limitations. In other cases when sensors used to perceive the machine environment are able to detect and localize the error, the executing element of the system obtains instructions to eliminate it. Today, the most advanced machine implemented in industrial assembly systems is an articulated robotic arm. A 6 degree of freedom (DOF) robot arm is capable of obtaining any given position and orientation in its workspace in a limited number of configurations. In recent years industrial robots with 7 DOF [5] have been introduced. These robots have the possibility of obtaining any position and orientation in their workspace in infinite different configurations as they possess a redundant kinematic chain. The robot arm is used to position the tool attached to the end effector to desired position in its workspace. Finally the robot end effector is responsible for solving the unpredicted situation successfully. Today, most sophisticated robot end effectors [6] compared to a human hand, which exhibits 27 DOF [7] including the arm, are simple and with limited capability. Therefore interference of human operators is imperative in some situations where the fault can be corrected only by human intervention. A traditional assembly cell has a delegated space where machines and robots work without any intervention. Regarding the safety regulations [8] the human operator and an industrial robot cannot share the same workspace.

However, today the number of applications where classical industrial robots are used as robot assistants is rising. A multimodal interaction scheme between industrial robots and humans is presented in [9]. By combing operator gaze, speech and use of soft buttons the operator is able to work in close collaboration with an industrial robot and participate in a hybrid assembly application. Authors in [10] address issues in humanrobot interaction and propose that by using proper sensors and equipment the interaction can be done with standard industrial robots. Current research applications suggest that the field of human-robot interaction utilizing industrial robots is gaining significance.

\section{MOTIVATION}

In the global market mass customization [11] has introduced tailored products for specific customer demands. A synergy and cooperation between human operators and industrial robots either as assistants or sophisticated tools is highly probable. Such hybrid assembly systems where humans work in close collaboration with robots pose certain benefits. Human operators will take advantage of robot speed and accuracy. On the other hand only the operator is able to perform certain complex manipulation and assembly 
tasks. Utilization of an industrial robot to do the tedious and repeating part of the work greatly alleviates the process for the human operator. Using the robot as a highly flexible and sophisticated tool (third hand) will most likely be a valuable application. A way for a human operator to communicate with a technical device is roughly divided into speech, gesture and touch. In this paper the last mentioned is implemented. As the robot is in interaction with a human safety needs to be considered first. A force-torque sensor on a robot arm measures forces applied only on the robot end effector. Measuring the current flow in robot motors will yield slow response times and insufficient sensitivity therefore posing a risk in applications. Vision systems can be used but there is a risk of blocking the vision system field of view. Measuring torques in robot motors [5] and adequate compliant behavior is possible. This technology is expensive and still in development but is suitable for human robot interaction. In this work a simple capacitive sensor design is proposed to aid human-robot interaction. The capacitive sensor will provide the robot with information of its close surrounding environment. By gaining proper information the robot can sense human presence and act accordingly.

\section{THE CAPACITIVE SENSOR}

The capacitive sensor is able to measure the change of capacitance with respect to distance of human body. It uses human body capacitance as an input. For this technical solution an Arduino microcontroller [12] and its capacitive sensing library has been used. This library converts two or more of Arduino's pins into a capacitive sensor that senses capacitance of a human body. The capacitive sensing library works by changing the microcontroller send pin (pin 4) to a new state and then waits for the receive pin (pin 2) to change to the same state. A variable in the program is timing the receive pin state change and reports the variable's value which is monitored on the serial port. The system requires a high value resistor connected between the send and receive pins, and a capacitive sensor as shown in Fig. 1.

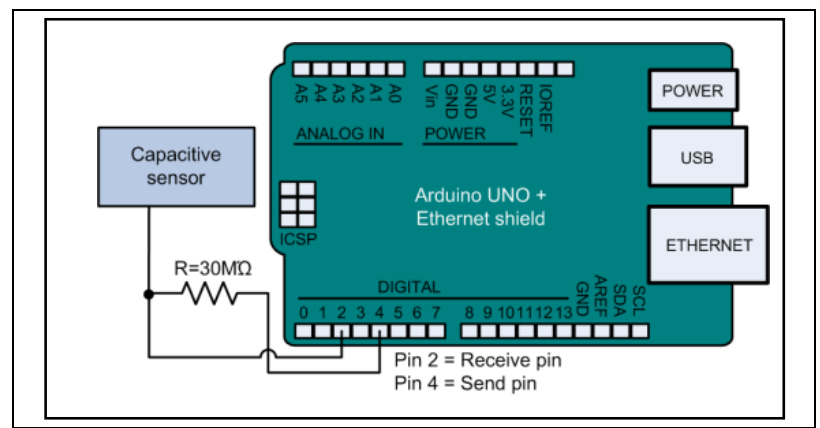

Fig. 1. Schematic view of the Arduino board and capacitive sensor

We used two different capacitive sensor setups as shown in Fig. 2. The four layer sensor has two layers of rubber foam, a layer of copper foil, and a layer of metal mesh. Copper foil layer is grounded and used to minimize eventual stray capacitance from the system, while the metal mesh is accounted for capacitance sensing. A rubber foam layer separates two conductive layers from each other and from the robot arm. In the three layer design there is no grounding plane and the sensor is smaller in size $(15 \times 15 \mathrm{~cm})$. When mounted on the robotic arm the capacitive sensor may in some cases pick up stray capacitance from the environment. This stray capacitance increases initial sensor untouched capacitance, and that interferes with preset thresholds. To minimize the influence of the environment, the design of the capacitive sensor is of most importance. Furthermore, increase of sensor size has significant impact on gathering stray capacitance. Influence of the environment can be minimized using a grounded plane that runs close to the sensing plane.

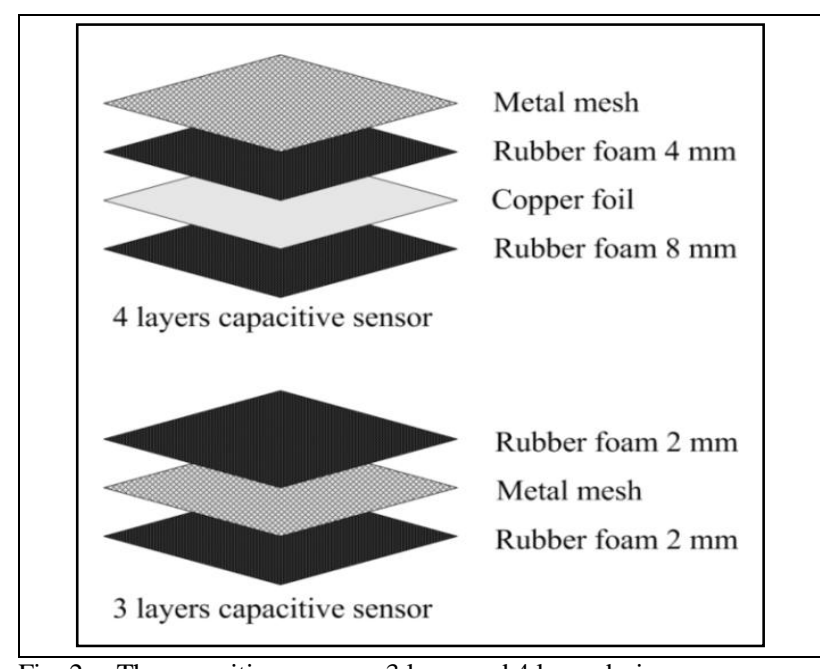

Fig. 2. The capacitive sensor - 3 layer and 4 layer design

\section{IMPLEMENTATION AND APPLICATION}

In the developed application the human operator is able to stop the robot arm by triggering the sensor. The capacitive sensor is mounted on the robot as shown in Fig. 3. The robot can suspend or slow down its motion. TCP/IP communication (socket messaging) between the Arduino and robot controller is established. Change in capacitance is measured by the Arduino controller and associated instructions are sent to the robot as shown in Fig. 4.

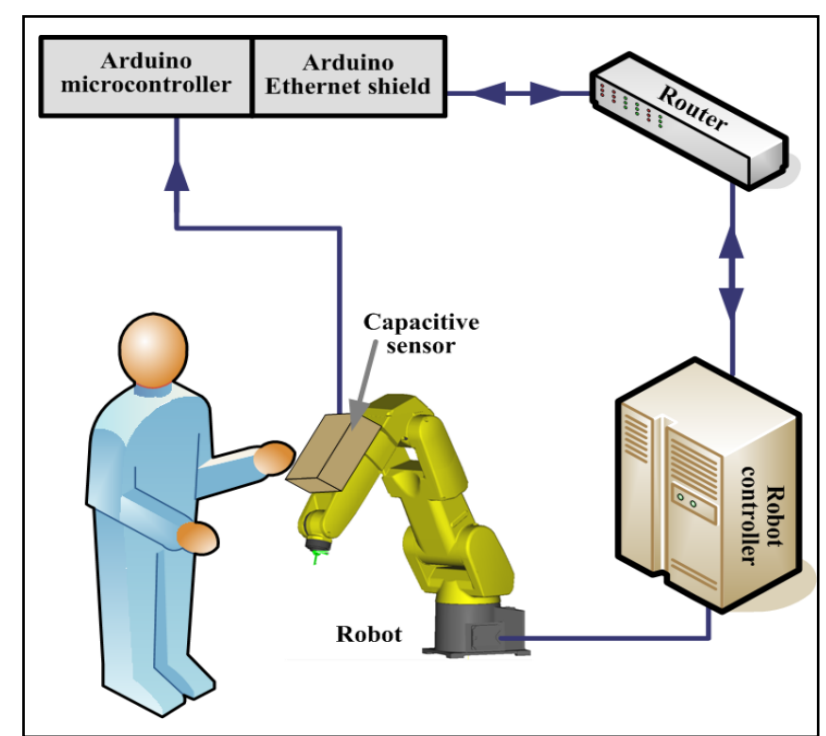

Fig. 3. Implementation of the capacitive sensor on a FANUC LR Mate 200iC5L robot arm 
In Fig. 4 a flowchart of a simple human-robot interaction application is depicted.

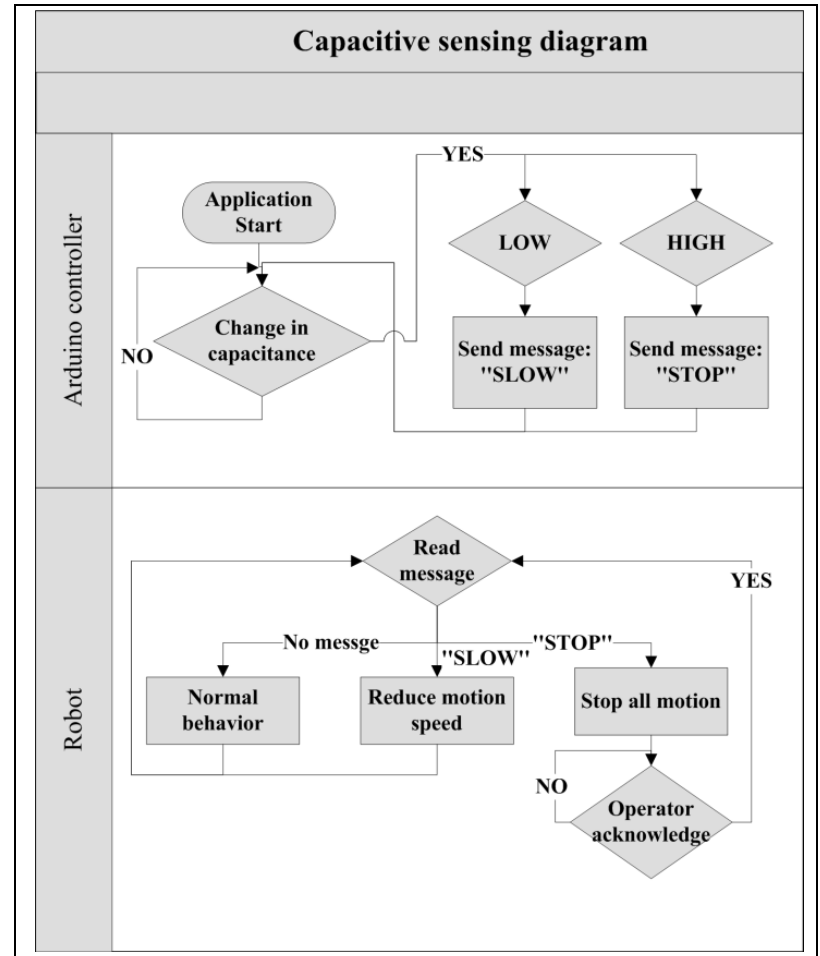

Fig. 4. Flowchart of a simple human-robot interaction application

\section{RESULTS}

Increase of measured capacitance is the most important aspect of the sensor output data. First tests of the sensor include a static scenario to test the difference in sensor designs (three and four layer). The sensor is placed on a table and change in capacitance is measured with respect to human hand distance. Different resistors were used with electrical resistivity of $10 \mathrm{M} \Omega, 20 \mathrm{M} \Omega, 30 \mathrm{M} \Omega$ and $40 \mathrm{M} \Omega$. In Fig. 5. results for the 3 layer sensor design $(15 \times 15 \mathrm{~cm})$ are presented, where in Fig. 6 are results for the 4 layer sensor $(40 \times 40 \mathrm{~cm})$.

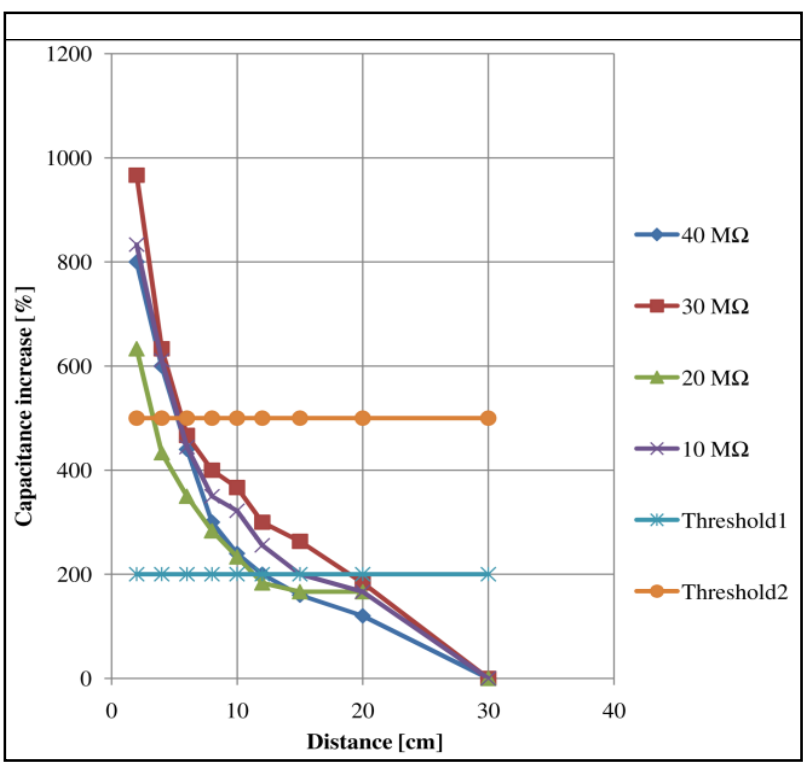

Fig. 5.Measured capacitance increase with respect to distance -3 layer sensor $(15 \times 15 \mathrm{~cm})$

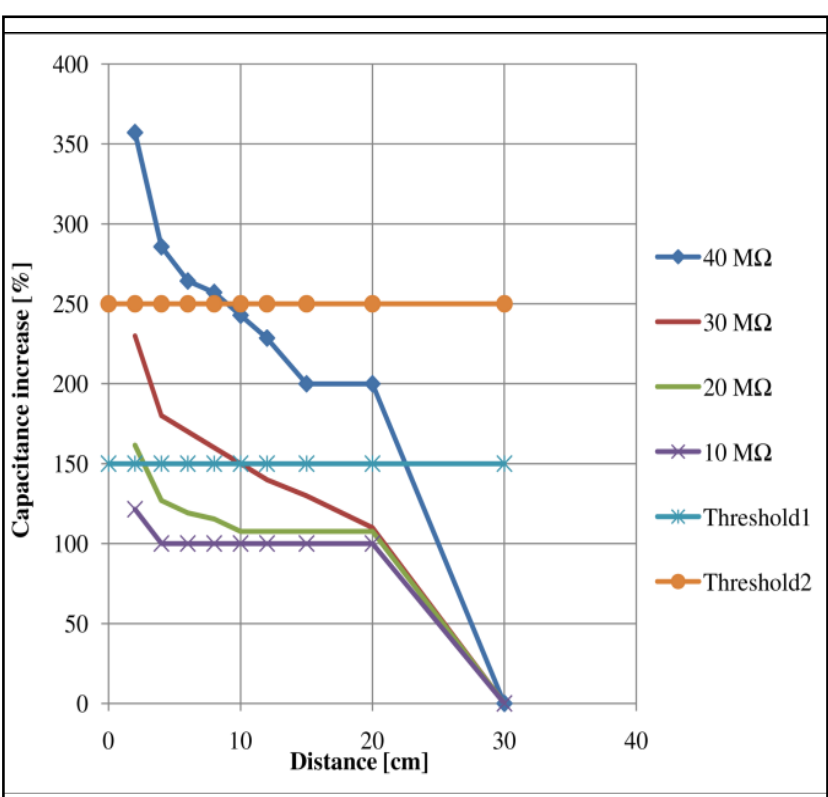

Fig. 6.Measured capacitance increase with respect to distance -4 layer sensor $(40 \times 40 \mathrm{~cm})$

The increase of measured capacitance is critical in application where the operator will use his hand to stop or reduce the speed of the robot.

The two horizontal lines suggest the threshold capacitance range where the robot can slow down (between Threshold1 and Threshold2) or stop its motion (above Treshold2). Any increase of capacitance below Threshold1 will not trigger any instructions for the robot. The smaller sensor (3 layer) gave better results regarding the capacitance change and was used in later experiments on the robot arm. As the readings from the capacitive sensor have a certain noise higher thresholds were used. All data in Fig. 5 and Fig. 6. are averaged from 10 measurements. The measured capacitance for identical case scenarios roughly differ by a maximum of $70 \%$.

Tab. 1 shows the increase of measured capacitance while the hand is contact with the sensor. The increase is between 5-10 times higher compared to hand distances of $2 \mathrm{~cm}$.

\begin{tabular}{|l|c|c|c|c|}
\hline & $40 \mathrm{M} \Omega$ & $30 \mathrm{M} \Omega$ & $20 \mathrm{M} \Omega$ & $10 \mathrm{M} \Omega$ \\
\hline Small sensor & $4843 \%$ & $5045 \%$ & $4541 \%$ & $5606 \%$ \\
\hline Large sensor & $4324 \%$ & $2523 \%$ & $1708 \%$ & $721 \%$ \\
\hline
\end{tabular}

Tab. 1.Measured capacitance increase when touching the sensor

While the sensor is mounted on the robot a dynamic situation occurs. The operator needs to stop the robot before it gets into contact with his body. The 3 layer sensor design with electrical resistivity of $30 \mathrm{M} \Omega$ and size $15 \times 15 \mathrm{~cm}$ was used. In the test scenario the robot is moving with continuous speed toward the human operator. Several speed tests have been conducted and are presented in Fig. 7. The operator uses his finger, hand, arm or whole body to stop the robot. As each part of the body induces different amount of capacitance the data significantly differs. 


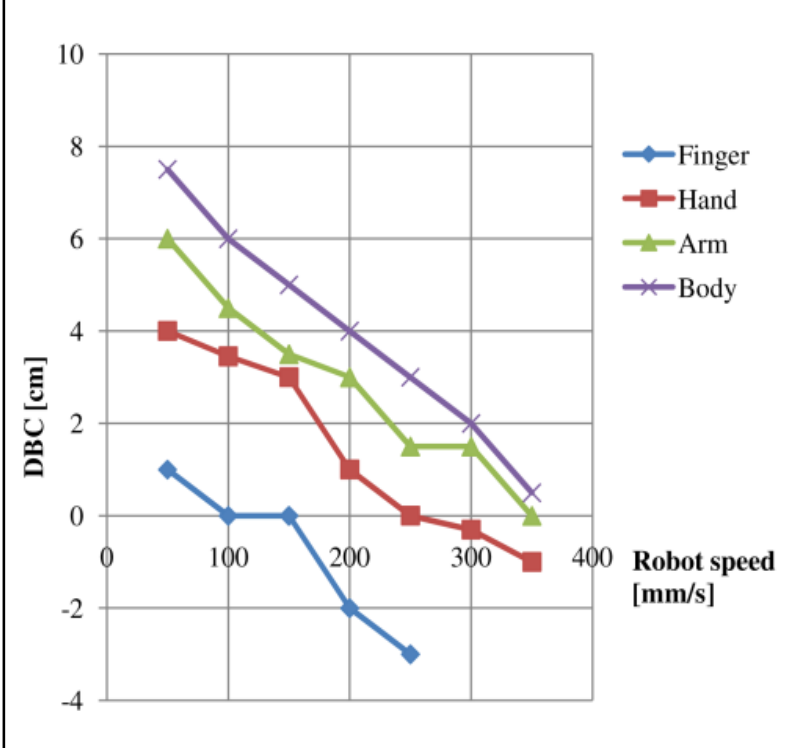

Fig. 7. Distance before contact (DBC) with respect to robot speed and different obstacles - human finger, hand, arm and body

Distances of $0 \mathrm{~cm}$ are soft touches of the robot where collision was almost avoided. Negative distances suggest that the robot penetrated into the operator safety zone. This was measured by using an elastic layer behind the capacitive sensor. Distance before contact (DBC) is acceptable for speeds lower than $300 \mathrm{~mm} / \mathrm{s}$ when the operator is stopping the robot using his arm and body, or lower than $250 \mathrm{~mm} / \mathrm{s}$ using his hand. Any higher speed could either result in collision with the operator hand or with unacceptable clearing distances. Trying to stop the robot using only a finger induces a very small change in capacitance.

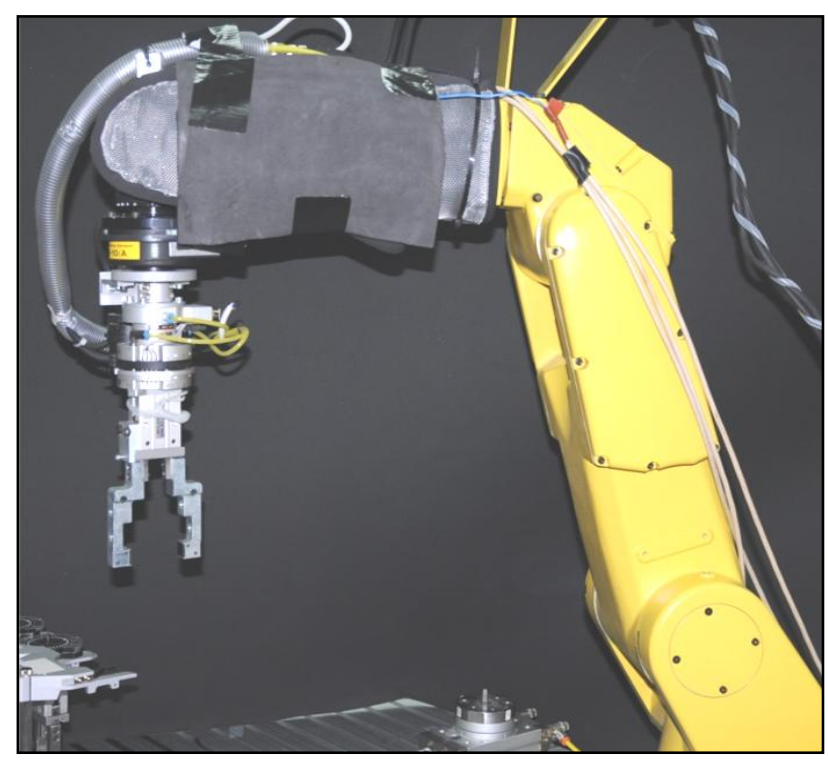

Fig. 8. The capacitive sensor

The data shown in Fig. 7. reflects the tests done on experimental setup with a small industrial robot as shown in Fig. 8. (FANUC LR Mate 200iC 5L) with cycle time of $4 \mathrm{~ms}$. In case of implementing the capacitive sensor on a larger robot with different dynamic characteristics the maximum safety speed can differ.

\section{CONCLUSION AND FURTHER WORK}

A simple capacitive sensor has been developed and its implementation in a human robot interaction scenario was addressed.

The capacitive sensor still has limitations regarding its size and residual capacitance. This issue will be solved using different electronic architectures and combination of different layer designs in future applications.

The developed capacitive sensor can be used on any architecture which supports socket messaging. Regarding connectivity, the Arduino microcontroller can send digital signals to any hardware as $24 \mathrm{~V}$ or $0 \mathrm{~V} \mathrm{DC}$. By this means any industrial hardware can be connected to the capacitive sensor controller. Using sensor fusion where an industrial robot can hold several sensors will allow more precise localization of the human operator in the robot work area. By this means the operator can roughly hand guide the robot toward any direction.

In conclusion the developed capacitive sensor mounted on a small industrial robot provides satisfactory results in human-robot interaction. As long as robot speed is not exceeding $250-300 \mathrm{~mm} / \mathrm{s}$ human safety is not at risk.

\section{REFERENCES}

[1] Frei R., Serbanuta T.F. \& Di Marzo Serugendo G. (2012). SelfOrganising Assembly Systems formally specified in Maude. Journal of Ambient Intelligence and Humanized Computing, pp. (1-20), ISSN 1868-5137

[2] Di Marzo Serugendo G. \& Frei R. (2012). Self-awareness in Agile Assembly Systems. Available from: http://www.awarenessmag.eu Accessed: 2012-09-01

[3] Stipančić T., Jerbić B. \& Ćurković P. (2011). A Robot Group Control Based on Bayesian Reasoning, Lecture Notes in Engineering and Computer Science, Volume 2191, S. I., Gelman L., Hukins D. W., Hunter A., Korsunsky A. M. (Ed.). Hong Kong Newswood Limited, 2011. pp. (1056-1060)

[4] Svaco M., Sekoranja B. \& Jerbic B. (2011). A Multiagent Framework for Industrial Robotic Applications, Cihan H. Dagli (Ed.) Proc. of the Complex Adaptive Sytems Conf., Chicago, pp. (337-342), Elsevier B.V.

[5] Bischoff R. (2009). From Research to Products. The Development of the KUKA Light-weight Robot," 40th International Symposium on Robotics, Barcelona, Spain

[6] Alan S. Brown (2008). Why Hands Matter, Mechanical Engineering, Vol. 130, No. 7

[7] Agur A. M. R., Lee M. J. (1999). Grant's Atlas of Anatomy, Lippincott Williams and Wilkins, ISBN 978-0683302646

[8] Ogorodnikova O. (2006). Human-robot Interaction Safety Problems, RAAD'06, Proceedings of the 15th International Workshop on Robotics in Alpe-Adria-Danube Region, Balatonfured

[9] Bannat A., Gast J., Rehrll T.,Rosel W., Rigoll G. \& Wallhoff F. (2009). A Multimodal Human-Robot-Interaction Scenario: Working Together with an Industrial Robot J.A. Jacko (Ed.) Human-Computer Interaction, Part II, HCII 2009, LNCS 5611, pp. (303-311)

[10] Giuliani M., Lenz C., Müller T., Rickert M. \& Knoll A. (2012) Design Principles for Safety in Human-robot Interaction. International Journal of Social Robotics, 2(3), pp 253-274, September 2010

[11] Hales H. L. (1992). Automating and Integrating the Sales Function: How to Profit From Complexity and Customization. Enterprise Integration Strategies, Vol. 9, No. 11, pp (1-9)

[12] http:Ilwww.arduino.cc Accessed on: 2012-06-12 